\title{
MAS Coordination Strategies and their Application in Disaster Management Domain
}

\author{
Vivek Kumar Singh $^{1}$, Neelam Modanwal ${ }^{2, a}$, Swati Basak ${ }^{2, b}$ \\ ${ }^{I}$ Department of Computer Science, South Asian University, \\ New Delhi, India \\ 1vivek@cs.sau.ac.in \\ ${ }^{2}$ Department of Computer Science, Banaras Hindu University \\ Varanasi, India \\ ${ }^{a}$ neelam.bhu1@gmail.com \\ bwati.basak.bhu@gmail.com
}

\begin{abstract}
Multi-agent System (MAS) formulations have been developed and explored in the past in a variety of domains, ranging from optimization problems to game theory. However, there are certain issues that are common across all the domains. Cooperation and coordination of agents in MAS is one such important area. In this paper, we have first presented a very brief survey characterizing the cooperation and coordination strategies that have been frequently used. Thereafter the paper progresses to explore the applicability of MAS formulations in disaster management domain. We have identified the characteristics and requirements of disaster management domain; described the ways in which MAS formulations can be used in this domain; and presented the preliminary results of our simulation experiment of a greedy approach to disaster recovery.
\end{abstract}

Keywords- Multi-agent Systems, Coordination, Cooperation, Disaster Management.

\section{INTRODUCTION}

A Multi-agent System (MAS) is comprised of multiple interacting intelligent agents. Agents in the MAS could all be of same type (homogeneous) or different (heterogeneous). Homogeneous MAS formulations employ agents that have similar capabilities and are often designed by a single individual. Heterogeneous agents, on the other hand, are usually designed by different individuals and agents in it may have different goals. An agent in a MAS may represent anything from a software program to a robot (or a even a human actor). Agents are autonomous, may be reactive or proactive, and have the ability to communicate with other agents. MAS are used to solve problems which are either difficult for an individual agent to solve or when the problem is inherently comprised of multiple actors interacting together. Over the past few years MAS formulations have been designed and explored across a variety of domains such as e-commerce, autonomic computing, scheduling, modeling social processes and structures, game theory, energy management, distributed computing, micro-grid system etc.

MAS formulations employ a large number of agents which interact with each other in various ways. If the agents in MAS are working together to solve some complex problem, they will possibly cooperate or coordinate with each other to achieve the global solution. Sometimes agents, not explicitly coordinating their activities, may agree to form coalitions. Coalitions may be formed either to maximize either the system-wide gain or payoffs of individual agents. On the other hand, in certain situations agents may also compete with each other for resources. Coordination is a however a central theme of interaction in almost every MAS formulations. A number of strategies have been advocated by MAS designers to achieve coordination in MAS. This includes task sharing structure used in centralized formulations, distributed contract net protocol, coalitions and other game theoretic approaches.

MAS formulations, after being successfully used across a large number of domains, are now being explored for possible use in disaster management domain. In fact there are two ways in which MAS formulation can be used in disaster management domain: (a) as a multi-agent information system situated in the environment, that can help both in needs assessment and also in coordinating disaster response activities; and (b) as a simulation toolkit to explore and evaluate the effectiveness of various disaster management activities in a virtual setting. Both these uses are however interrelated. For example, in an attempt to design a virtual simulation, a designer may also come up with an efficient information communication system that can help in recovery in actual disasters. Disaster situation pose peculiar characteristics that makes the task of designing MAS formulation very challenging. In this paper, we have first surveyed (Section II) the coordination strategies used in MAS formulations in the past, followed by identifying the key characteristics of disaster domain and the design requirements thereof (Section III). We have performed a MAS simulation experiment for disaster recovery strategy and presented our preliminary results in Section IV. The paper concludes (Section V) with a brief discussion of the results, and relevance $\&$ potential of the work. 


\section{COORDINATION IN MAS}

A MAS formulation employs many agents to achieve a goal. The individual agents in such a system may be homogeneous or heterogeneous in their capabilities. However, irrespective of the nature of MAS design coordination among agents in MAS is always a core issue. Coordination and cooperation allows the agents to manage their interdependencies and the type and nature of interactions. Coordination and cooperation differ in degree of inter-agent knowledge and beliefs. Depending on the type of MAS formulation, different schemes of coordination and cooperation may be used. Cooperation is generally seen when agents in a MAS are directly aware of each other and work towards a common goal. Coordination is a bit broader and may be there even in situations in which agents are not directly aware of each other and yet coordinate their activities indirectly. Cooperation and coordination strategies in MAS have been extensively explored in the past. MAS designers often take inferences from research on coordination in the diverse disciplines of social sciences, organization theory, game theory and even biological systems. Coordination and cooperation between agents in MAS essentially implies management of interdependencies between agent's activities and integrating them with distributed heterogeneous sources [1], [2], [3].

Cooperation can be of two types: implicit and explicit. Cooperation is usually observed when the actions of each of the agents satisfy either or both of the following conditions: (a) the agents have a (may be implicit) goal in common, which no agent could achieve in isolation; and (b) the agents perform actions which achieve not only their own goals, but also the goals of agents other than themselves. Coordination refers to the process by which the individual decisions of the agents result in joint decisions for the group. To cooperate successfully, each agent must maintain a model of the other agents. Coordination, being a process of acting together, can be accomplished by both intentional and non intentional agents. Coordination and cooperation processes \& mechanism vary with the kind of MAS formulation used, namely centralized and distributed.

\section{A. Coordination in Centralized Formulations}

In a centralized MAS formulation there is usually a central agent which is responsible for command and control functions. This type of formulation is generally used when there is a complex problem which can be divided into smaller sub problems. The coordination in this kind of formulation encompasses three different phases: dividing the problem into sub problems, solving the sub problems and then combining the results of the sub problems to obtain the global result [4], [5]. In the first phase of dividing the problem into sub-problems the central agent analyzes the problem and divides it into smaller sub-problems. Quite often the division follows a hierarchy where first level subproblems can be further sub divided into smaller independent sub-problems, and the process continues until the sub problems are of an appropriate granularity to be solved by individual agents. The different levels of division represent different levels of problem abstraction. The central agent is responsible for the divisions and subsequent assignments. During the second phase the identified sub-problems are solved. Since, the sub problems are parts of a bigger problem, there may be various interdependency relationships that need to be addressed. The central agent coordinates in this sharing of information between agents. In the third phase, the solutions of sub problems are combined (integrated) together to constitute the global solution. The partial solutions may require combining at different levels of abstraction. The coordination and cooperation in centralized formulations is thus a process of transferring and assigning decision making process to the higher levels of the organizational hierarchy.

\section{B. Coordination in Distributed Formulations}

Another MAS formulation that is quite frequently used in a number of situations is distributed structure. Here, no central agent is present and hence the agents need to coordinate their activities in an independent and distributed manner. The control decisions are distributed across the agent population. Since there are no designated leaders, agents in MAS have to work out all issues in the problem solving (identifying the problem, dividing it into sub problems, scheduling the sub problems and combining the solutions) in a decentralized distributed manner. Coordination in this case requires more and varied communication than that of centralized formulations. The Contract Net (CNET) protocol [6], [7] is one such high-level protocol that allows distributed coordination of agents in a MAS. The CNET scheme uses following sequence of activities: (a) identifying the problem by an agent and dividing it into sub problems, (b) the initiator agent announces the task and requests agents in the population to bid for it, (c) individual agents process task announcement and decide whether they are eligible for the task and that if they would like to bid for a task, (d) the bids are then processed by the original agent, (e) finally tasks are awarded usually to the lowest bidder. The successful bidder then proceeds to solve the problem assigned to it and it may involve generating new sub-tasks. Distributed coordination schemes [8] are better when either it is not known a priori how problem can be divided into sub problems and possibly no agent has the necessary know how to act as leader, or when the environment is inherently distributed.

\section{Comparing the Coordination Schemes in MAS}

Centralized task structure based coordination schemes have the advantage that it is more efficient to manage multiple agents working towards a common goal. Moreover, it requires less communication and the solutions are often obtained within reasonable computational cost and time. An obvious disadvantage is that the quality of solution depends on how the central agent divides the original problem into various sub problems. If the central agent does not have a clear picture of the sub tasks required to solve the problem, the formulation may not produce good results. The second disadvantage to centralized planning is that too many resources could be channeled to solve a problem which may be wrongly conceived by the central agent. The distributed 
coordination scheme, on the other hand, has the advantage that the individual agents have more freedom and flexibility in choosing their own strategy to solve a task. However, distributed schemes incur more communication overhead.

\section{DiSASTER MANAGEMENT DOMAIN}

Disaster management has become a very important area worth exploration in the last few years, primarily due to the large number of disasters occurring across the world. Disaster management involves coordinating a large number of emergency responders to rescue people and recover infrastructure. The environment in which the responders act is often hazardous and full of uncertainties. Disaster response requirements are often identified by several distinctive characteristics and factors relevant to managing them such as: (i) disaster overwhelms the available resources, (ii) disaster response should be immediate, (iii) disaster events are unpredictable, (iv) uncertainty and incompleteness of information \& resources, (v) need for information \& communication, and (vi) disaster recovery strategy to recover from secondary crisis. Disasters, both natural and man-made, can strike anytime or anywhere. There are two ways to overcome disasters: the first is to prevent them from occurring, and second to have an emergency system and plan of operation prior to the occurrence of any crisis. In either approach, information technology plays an important role in crisis management. Disasters, like earthquakes, floods, etc. can cause large number of deaths, injuries and homeless people. Appropriate responses are needed in the form of allocating resources to handle the effects of disasters.

A number of full scale disaster response systems have been developed in the recent past. Some of the important ones include ALADDIN [9], RoboCup Rescue [10], DrillSim [11], DEFACTO [12], and FireGrid [13]. The DrillSim, DEFACTO, FireGrid and ALADDIN systems focus on studying fire evacuation crisis, whereas RoboCup Rescue focuses on urban search and rescue operations. Almost all current systems aim to roughly support the response activities with very small interest on improving the effectiveness of response operations. A disaster response system should take in consideration the domain requirements to increase response effectiveness.

During the last few years, the scientific community has also reported several IT solutions to deal with communication, coordination and decision making challenges in large-scale disaster management system (e.g. tsunamis, hurricanes or terrorist attacks). The proposals range from those focused on the communication and notification [14], [15], [16], [17], [18], [19] to those focused on information management, resource allocation and decision making [20], [21]. Some examples of these systems are CATS [22], OpenGIS [23], DERMIS [24], Sahana [25], DUMBONET and Eplan [26]. Solutions focused typically involve the communication infrastructure (e.g. WiMax mobile) and usually satellite communication. Although they provide strong communication support in the affected area, they require expensive equipment and supporting infrastructure, e.g., mobile antennas deployed on communication trucks. The information collected by emergency management systems is typically used to make decisions and coordinate the first responders' activities. These systems are particularly useful to coordinate large disaster relief efforts, but as stated earlier they also require strong communication support.

In a recent work [27] a domain-independent agent architecture was proposed that addresses real-time constraints, available network resources and the need to coordinate and cooperate with problem-solving activities of other agents. It contains five components local agent scheduling, multi-agent coordination, organizational design, detection and diagnosis and on-line learning. These are designed in such a way that a range of different situationspecific coordination and cooperation strategies can be implemented and adapted as the situation evolves. The application, named MobileMap (mobile collaborative application) for reducing radio based communication in between the firefighters. MobileMap allows ad hoc communication, decisions support and collaboration among firefighters in the field using mobile devices. This solution complements the radio communication systems. Since the interactions supported by MobileMap are recorded, it is possible to analyze such information after the crisis. This application was initially designed to help firefighters to arrive faster to the emergency site, to allow them to exchange digital information during emergency response processes and to reduce the need for radio communication. However, the current version of the tool also supports the decision making process carried out by firefighters in the rescue place or disaster place and the emergency learning process (after the crisis). The tool was tested in simulated and real emergency scenarios. The obtained results demonstrated that the firefighters believe that MobileMap helps reduce the arrival time to the emergency place (crisis place). The application could also help reduce the time required to control the emergency, which typically increases the protection to human life and property from any type of crisis.

Typically the disaster management system can be viewed as a four phase process [28]. The first phase is disaster assessment in which the losses and their magnitude are identified. Second phase involves needs assessment where the resource requirements for recovery from disaster are assessed. The third phase is prioritization of response resources. This is often necessary as resource availability often does not match with the needs. The fourth phase is actual response in which the crisis resources are deployed. Taking into account the dynamic and uncertain nature of disaster situation, the disaster recovery methodology must incorporate tools for data fusion, resource management, coordination of actors, adaptation to environment changes, distributed decision making and decentralized control. The progress in information technology and agent modeling languages \& techniques has paved the way for use of agent formulations in disaster management domain. There can be two ways in which agent formulations can be employed in disaster management domain. One is to use agent based information systems to help with the information needs and 
activity coordination in actual disaster recovery process. The other way could be to simulate virtual disasters and then experiment with different disaster recovery strategies. The simulated experiment can be used to evaluate the effectiveness of strategies for data fusion, resource management, coordination \& control of actors. The inferences obtained from simulation experiments can be used to modify actual disaster recovery strategies and frame new ones.

\section{EXPERIMENTAL WORK}

We have designed an agent based simulation for disaster situation. The main aim of the simulation design is to explore and evaluate effectiveness of different disaster response strategies in a virtual setting. We implemented our simulation using NetLogo [29]. Broadly two different kinds of disaster response coordination strategies may be implemented: (a) centralized coordination approach, and (b) distributed coordination approach. The problem in disaster situation is to rescue a large number of people and often to recover some infrastructure. This is to be achieved in an uncertain and hazardous environment, where enough means of communication may not be available. Moreover, the resources available are often much less than that required for the rescue and recovery operations. One more important concern is the time constraint associated with recovery operations. In certain situations if rescue operations are not initiated and completed in time there can be huge loss to life and property. We have implemented a simple model to simulate a disaster scenario, where the landscape is modeled as a grid and more than one site on a grid may be in a disaster situation. The rescue and recovery infrastructure is positioned at random locations on the grid.

\section{A. Model Setup}

We devised a 50 X 50 grid in NetLogo. The key entities in the model are represented by patches and turtles (agents in NetLogo). The patches represent stationary landscape on the grid and turtles represent movable parts like people, rescue workers, medical professionals, ambulance, fire tenders etc. We randomly selected three patches on the grid and constructed disaster area around these patches. The size and magnitude of each of the disaster areas are kept different. Depending on the size and magnitude of a disaster, initial values for persons and infrastructure affected is set. For example, a bigger disaster zone will have more people wounded than a smaller disaster zone. The rescue and relief resources are also positioned on the grid and values for them are set to deliberately keep the resource availability lesser than that required to satisfy the needs of all the disaster zones simultaneously. The rescue agents included a team of doctors, nurses and relief workers. The recovery agents included ambulance, fire tenders and municipal vehicles. Distances in the model are calculated by assuming two adjacent patches being one unit of distance away from each other. All computations regarding time required for a rescue or relief worker to reach to a disaster zone from its depot is thus calculated accordingly.

\section{B. ModelParameters \& Simulation Runs}

We aimed to explore the effectiveness of alternative coordination and cooperation strategies in disaster response task. The first setting that we explored is a task structure based centralized formulation. This is often the most commonly used strategies in real disaster situations. We assume that there exists a central disaster management centre which is responsible for coordinating the activities of different entities involved in disaster response. The disaster manager thus communicates and coordinates with different establishments such as hospitals, fire stations and municipality for resource scheduling. It is the disaster manager which has to asses the needs of a disaster site, prioritize the requirements of different disaster sites, assign different resources to sites, and monitor the response process. Once it gets information about the scale and magnitude of a disaster at a site, it works out a needs assessment taking into account the approximate values it obtained in the communication. When more than one disaster information is received and the needs assessment is higher than the resources available, scheduling priorities have to be set.

The disaster manager in our model takes a greedy approach in assigning resources. The resource requirements of a disaster site are satisfied by assigning a major part of the resources from the nearest resource centre (such as the nearest hospital). In case the resources available there are inappropriate, the disaster manager tries to ensure resource availability from the other nearest resource centre. Once a resource is assigned to cover a disaster site, it moves to the site. However, it takes some time for a set of rescue workers to reach a disaster site. This time is calculated by using the grid distance. The problem is that there is a severe time constraint associated with successful rescue and recovery. We modeled this through assigning a time of death to the wounded persons as well as other persons such as those who may die due to unavailability of food or other help. The aim in disaster response is therefore to maximize the recoveries in as low as cost as possible.

\section{Results}

We made multiple runs of the model with varying parameter values. The disaster site placement, its scale and magnitude, number of resources available and times to death for wounded and other suffering people have all been varied. We used certain simple assumptions that a doctor may attend some $x$ number of persons at a time and similarly a rescue worker may attend some $y$ number of persons at a time. We did not vary the values of $\mathrm{x}$ and $\mathrm{y}$ across runs as it hardly adds any significance to the results. Further, we have assumed that a doctor (and other rescue workers) are capable of taking certain independent decisions. For example, a doctor scheduled to visit a place will also try to help any other suffering person nearby. Similarly other rescue workers are also scheduled by the central manager to attend to a specific disaster site. This includes relief workers, municipal vehicles etc. We obtained interesting results. Figures 1 and 2 present a snapshot of two different runs. 


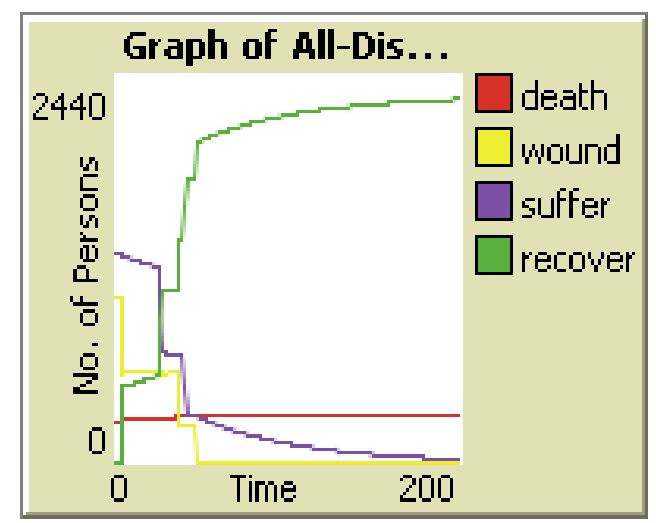

Fig. 1 A sample run with a total population of 2600 peoples affected. Total time to recover for three disaster sites is $71,44 \& 154$.
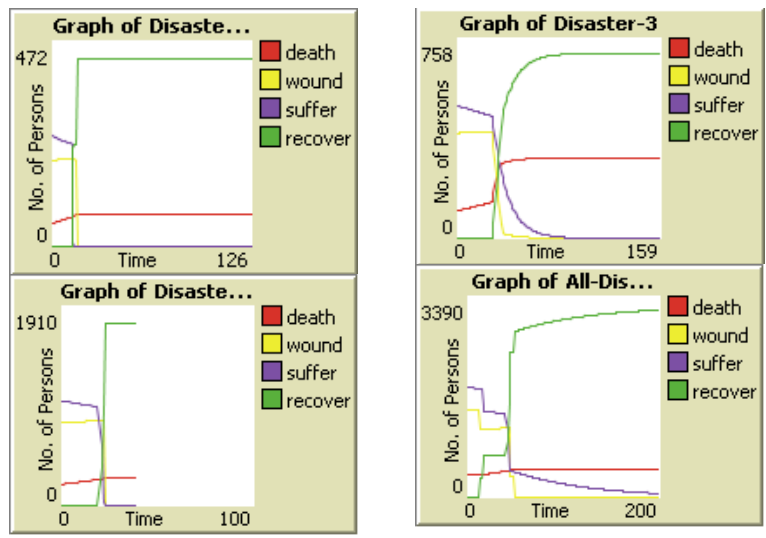

Fig. 2 A sample run of the model with the affected populations in three sites being 500, 2000, 1000. Top left is for disaster site 1, bottom left for site 2, top right for site 3 and bottom right the combined result. The times to recovery are $126,30, \& 158$.

The results obtained demonstrate that as the rescue resources reaches a disaster site, the number of deaths decrease sharply. Those who are wounded are attended and hence their number also decreases. What is however more important is that the time to recovery and the point from which the decline in number of sufferers start, varies a lot across different disaster sites. The main reason for this is the greedy assignment of resources. Those sites that have sufficient number of resources available nearby, recover quickly. But the sites that are either farther from resource centers or for whom the number of resources available locally are less than sufficient, the time and rate of recovery is relatively poor. Further, due to greedy nature of rescue operations, the disaster response prioritization may not be optimal. These results are however just one part of the picture. It remains to be seen what happens with other disaster response strategies. The crux of the matter however is that simulation studies can be a good tool to experiment and evaluate different disaster response strategies. A good amount of work in this area has started recently and many valuable results are being obtained.

\section{CONCLUSION}

Coordination and cooperation are two key issues in Multi-agent Systems. Various applications of MAS formulations in different domains have devised different strategies for achieving coordination and cooperation. These strategies are broadly classified into centralized and distributed schemes. With growing use of MAS formulations in newer domains, it has become more important to evolve suitable strategy for achieving coordination and cooperation. A scheme may work suitably in a domain but may not be optimal for a different kind of domain. Moreover, an altogether new scheme may be required for a novel domain. More exploratory work is necessary to evaluate the applicability of different coordination strategies across domains.

We have explored the use of a coordination strategy in disaster response domain. The disaster response environment is full of uncertainties and the response requirements are often identified by several distinctive characteristics such as: (i) disaster overwhelms the available resources, (ii) disaster response should be immediate, (iii) disaster events are unpredictable, (iv) uncertainty and incompleteness of information \& resources, (v) need for information \& communication, and (vi) disaster recovery strategy to recover from secondary crisis. All disasters are unique in these characterizing features. Therefore, a scheme to coordinate the response activities may not be designed a priori. Emergency responses vary across different disasters and every disaster calls for a unique (not a fixed a priori design) coordination strategy. However, designing a dynamic strategy that generates plans according to a disaster characteristic is a challenging task and requires more exploratory work on coordination strategies in disaster management. We have experimented with one such simple design strategy where we evaluated a task structure based coordination scheme for managing responses to a disaster situation with varying scale and magnitude. The results demonstrate that the scheduling and coordination scheme design has a huge impact on the disaster recovery. Those sites that are attended earlier result in more successful rescues than the other ones. A good response strategy therefore should collect appropriate amount of information before making scheduling decisions. Moreover, a good scheme to coordinate the rescue activities of workers is also a major role player in better disaster response. The taskstructure based coordination we used is just one of the possibilities; other schemes, particularly distributed ones, also need to be explored.

\section{REFERENCES}

[1] G. Weiss, "Multi-Agent Systems - A Modern Approach to Distributed Artificial Intelligence", MIT Press, 2001.

[2] M. Wooldridge, "An Introduction to Multi-Agent Systems", Reprint, John Wiley \& Sons, 2002. 
[3] N.R. Jennings, S. Katie \& M. Wooldridge, "A Roadmap of Agent Research and Development" In Proc. AAMAS, 1996.

[4] G. Agha, "ACTORS: a Model of Concurrent Computation in Distributed Systems"; MIT Press, Cambridge, 1986.

[5] R. Davis, "Report on the workshop on Distributed AI", ACM SIGART Newsletter, 1980.

[6] R. Smith, "The contract net protocol: high level communication and control in distributed problem solver". IEEE Transactions on Computers, 1980.

[7] R.G. Smith, "The Contract Net: a formalism for the control of distributed problem solving"; In Proceedings of the 5th International Joint Conference on Artificial Intelligence, 1977.

[8] P. Sousa \& C. Ramos, "A distributed architecture and negotiation protocol for scheduling in manufacturing systems"; Computers in Industry, 1999.

[9] N.M. Adams, M. Field, E. Gelenbe, D.J. Hand, N.R. Jennings \& D.S. Leslie, "The ALADDIN Project: Intelligent Agents for Disater Management", IARP/ EURON Workshop on Roboitics for Risky Interventions and Environmental Surveillance, 2008.

[10] RoboCup Project, http://www.robocuprescue.org

[11] M. Balasubramanian \& V. Mehrotra, "DrillSim: A Simulation Framework for Emergency Response Drills", Proc. of ISCRAM, 2006.

[12] N.S. Marecki \& M. Tambe, "Agent Based Simulations for Disaster Rescue using the DEFACTO Coordination System", Emergent Information Technologies and Enabling Policies for Counter Terrorism, 2005

[13] D. Berry \& A. Usmani, "FireGrid: Integrated Emergency Response and Fire Safety Engineering for the Future built Environments", UK e-Science Program All Hands Meeting, Nottingham, UK, 2005.

[14] K. Kanchanasut et al, "DUMBONET: A multimedia communication system for Collaborative emergency response operations in disaster affected areas", International Journal of Emergency Management, 2007.

[15] A. Malizia et al, "SEMA4A: An ontology for emergency Notification systems accessibility", Expert Systems with Applications, 2010.

[16] B.S. Manoj \& A. Baker, "Communication challenges in emergency response", Communications of the ACM, 2007.
[17] A. Meissner et al, "Design challenges for an integrated disaster management communication an information system", In First IEEE Workshop on Disaster Recovery Network, New York, USA, 2007.

[18] S. F. Midkiff \& C. W. Bostian, "Rapidly Deployable Broadband Wireless Communications for Emergency Management", In National Digital Government Research Conference, Redondo Beach, CA, USA, 2001.

[19] C.P. Smith \& D. M. Simpson, "Technology and communications in an urban crisis: The role of mobile communications systems in disasters", Journal of Urban Technology, 2009.

[20] R. Chen et al, "Coordination in emergency response Management", Communications of the ACM, 2008.

[21] Y. Yuan \& B. Detlor, "Intelligent mobile crisis response systems", Communications of the ACM, 2005.

[22] J. Swiatek, "Crisis prediction disaster management", SAIC Science and Technology Trends II, 1999.

[23] J. Farley, "Building enterprise government using OpenGIS technology", In Proceedings of the open GIS Seminar, Geospatial Information and Technology Association, Charlotte, USA, 1999.

[24] M. Turoff, M. Chumer et al, "The design of a dynamic emergency response management information system (DERMIS)", Journal of Information, Technology Theory and Application, 2004.

[25] P. Currion, C. de Silva \& B. Van de Walle, "Open source software for disaster management", Communications of the ACM, 2006.

[26] Z. Zhang \& Q. Li, "An open urban emergency decision support system". In the International Archives of the Photogrammetry, Remote Sensing and Spatial Information Sciences, Beijing, China, 2008.

[27] A. Monares et. al., "Mobile computing in urban emergence situations: improving the support to firefighters in the field", Expert Systems with Applications, vol. 38, 2011.

[28] S.D. Ramchurn et. al, "Agent-based Coordination Technologies in Disaster Management- Demo Paper", Proc. of $7^{\text {th }}$ International Conference on Autonomous Agents and Multi-agent Systems (AAMAS), Portugal, 2008.

[29] U. Wilensky, "NetLogo", Centre for Connected Learning, Northwestern University, USA, 1999. 